SNUTP-94-60, hep-th/9407048

\title{
Abelian Chern-Simons field theory and anyon equation on a sphere
}

\author{
N. W. Park \\ Department of Physics, Chonnam National University, Kwangju, 500-757, Korea \\ Chaiho Rim and D. S. Soh \\ Department of Physics, Chonbuk National University, Chonju, 560-756, Korea
}

(July 7,1994$)$

\begin{abstract}
We analyze the Chern-Simons field theory coupled to non-relativistic matter field on a sphere using canonical transformation on the fields with special attention to the role of the rotation symmetry: $S O(3)$ invariance restricts the Hilbert space to the one with a definite number of charges and dictates Dirac quantization condition to the Chern-Simons coefficient, whereas $S O(2)$ invariance does not. The corresponding Schrödinger equation for many anyons (and for multispecies) on the sphere are presented with appropriate boundary condition. In the presence of an external magnetic monopole source, the ground state solutions of anyons are compared with monopole harmonics. The role of the translation and modular symmetry on a torus is also expounded.

03.70.+k,11.10.Ef,11.15.Tk
\end{abstract}

Typeset using REVTEX 


\section{INTRODUCTION}

The statistical property of quantum mechanical particles is known in two categories, fermion and boson. The dynamics of these particles is described systematically in secondquantized field theories. In two space dimension, however, anyonic statistics [1] is known to be possible. This observation has come not only from theoretical curiosity but also from experiment such as fractional quantum Hall effect [2]. One may even go further to define fractional statistics based on Hilbert space counting independent of space dimension [3].

The dynamics of anyon [4] 8 ] is described in the first quantized theory by introducing statistical gauge potential minimally coupled to the originally bosonic or fermionic theory. After singular gauge transformation (to make the singular gauge transformation is meaningful, one has to apply the so-called "hard-core" boundary condition), the statistical gauge potential can be removed and the wavefunction becomes multi-valued instead (statistical transmutation), from which the name anyon stems. The second quantized theory of anyons is yet to come [9]. Nevetherless, One can relate Chern-Simons (CS) field theory [10] in two space and one time dimension to the first quantized anyon theory: One can obtain the corresponding anyon equation using the second quantized field theory and Heisenberg equation 11.

The pure CS gauge action has no dynamical gauge field. By coupling the gauge field to an external source, one can investigate quantities such as Wilson loops. In fact, the correlators of Wilson lines in the non-abelian theory are related with the polynomial invariant of knot theory [12]. When the gauge field is coupled to a dynamical matter field, it plays the additional role of statistical gauge potential to the matter field, which is best seen in the abelian CS theory on an infinite plane [6-8]. Its non-abelian statistical property is also investigated using conformal blocks [13].

In quantizing the CS theory, some subtleties are noted due to the Gauss constraint. By solving the constraint first and quantizing the theory later, one may lose some information inherent to the quantized theory such as quantum holonomy [14]. In addition, restricting 
the Hilbert space to the one with definite particle number states and treating the number operator as a $c$-number [15, 16] arouse controversies [17]. On torus, this procedure makes the abelian CS coefficient rational which depends on the number of particles: The theory should pick up a certain coefficient to accommodate the $N$ particles. On the other hands, one can quantize the gauge field first and restrict the Hilbert space later according to the Gauss constraint (not necessarily onto the definite particle number states). If one identifies the fundamental domain of the torus with a rectangular unit cell and applies the periodic (translation) symmetry in the both direction [18], then one can treat the theory field-theoretically and does not need the abelian CS coefficient quantization.

The origin of the above discrepancy (except the quantum holonomy) may not be entirely due to the way of gauge field quantization. One can suspect that this comes from the conflict with the symmetry that the system is allowed to have (e.g. translation invariance and/or modular invariance), since the Gauss constraint (like in the presence of an external monopole source) can not be realized arbitrarily in general due to the inherent symmetry of the compact manifold. This paper is devoted to illuminate the role of the symmetry of the compact manifold (sphere and torus) on the CS field theory and its connection to the anyon equation. For this purpose, we start with investigating CS theory on a sphere field-theoretically following the method given in [18], which is simpler than the theory on a torus since harmonic one form is absent. (Related works can be found in [19,20] where the Gauss constraint is treated classically.) The same field-theoretic analysis can be used for the torus symmetry.

This paper is organized as follows. In section II we present the mode decomposition of the gauge field on two patches of the sphere. In section III we couple the non-relativistic matter field (fermion for definiteness) to the gauge field. The hamiltonian is re-expressed in terms of canonically transformed new field, which effectively solves the Gauss constraint. The expression of the constraint and hamiltonian is incorporated with the rotation symmetry, $S O(2)$ or $S O(3)$. The Hilbert space restriction due to the rotation invariance is investigated in detail. In section IV we present anyon equation for $N$-identical particles and 
for multi-species with the vanishing total charge. The boundary condition for the wavefunction with the rotation symmetry taken care of. In section $\mathrm{V}$ we introduce an external magetic monopole source and discuss its ground state and degeneracy. It is noted that the ground state solution of anyons is not annihilated by the ladder operator of $s u(2)$ unlike for the monopole harmonics. Section VI is the summary and discussion, where we discuss about the translation and modular symmetry on the torus and its consequences.

\section{TWO PATCH DESCRIPTION OF GAUGE FIELD}

The gauge field on a sphere is a singular function in general (connection on a $U(1)$ bundle). Therefore, one needs coordinate patches to describe the gauge field on the whole space [21]. We introduce two coordinate patches, which are two projected planes from the south and north poles respectively (FIG.1). The north (south) patch covers the whole points on the sphere except the south (north) pole. Let us denote a point as $\Omega=(\theta, \phi)$ on the sphere. The same point is described as $P_{N}=\left\{\vec{x}_{N}\right\}$ on the north patch $\left(P_{S}=\left\{\vec{x}_{S}\right\}\right.$ on the south), where

$$
\begin{aligned}
\vec{x}_{N} & =\left(x_{N}^{(1)}, x_{N}^{(2)}\right)=\left(\tan \frac{\theta}{2} \cos \phi, \tan \frac{\theta}{2} \sin \phi\right) . \\
\vec{x}_{S} & =\left(\cot \frac{\theta}{2} \cos (\pi-\phi), \cot \frac{\theta}{2} \sin (\pi-\phi)\right) .
\end{aligned}
$$

The two representations have a relation $\vec{x}_{S}=\left(-x_{N}^{(1)}, x_{N}^{(2)}\right) / \vec{x}_{N}^{2}$, which is in complex notation $z_{S}=-1 / z_{N}$ where $z=x^{(1)}+i x^{(2)}$. The metric $g_{\mu \nu}$ in either patch is diagonal and is given as

$$
g_{00}=1, \quad g_{i j}=-\left(\frac{2}{1+\vec{x}^{2}}\right)^{2} \delta_{i j}
$$

The Greek index stands for 3d component whereas the Latin for 2d. Some of the necessary ingredients for the description of the sphere is summarized in the Appendix for reference and notation convention. We will consider the physical quantity on one patch first (the north

patch for definiteness) and consider the other patch when necessary. Hereafter, we drop the subscript $N$. 
The Chern-Simons action for the gauge field $a_{\mu}$ is given as

$$
S_{g}=\frac{\mu}{2} \int d^{3} x \sqrt{g} \epsilon^{\mu \nu \rho} a_{\mu} \partial_{\nu} a_{\rho}
$$

where $d^{3} x \sqrt{g}$ is the invariant volume element and $\epsilon^{\mu \nu \rho}$ is the anti-symmetric Levi-Civita tensor. Let us introduce the (pseudo-)invariant $e^{\mu \nu \rho}=e_{\mu \nu \rho}$ with $e_{012}=1$, which is related with $\epsilon^{\mu \nu \rho}$ as

$$
\epsilon^{\mu \nu \rho}=\frac{1}{\sqrt{g}} e^{\mu \nu \rho}, \quad \epsilon_{\mu \nu \rho}=\sqrt{g} e_{\mu \nu \rho}
$$

using $g=\operatorname{det}\left(g_{\mu \nu}\right)>0$. Then the action is written as

$$
S_{g}=\frac{\mu}{2} \int d^{3} x e^{\mu \nu \rho} a_{\mu} \partial_{\nu} a_{\rho}=\frac{\mu}{2} \int a \wedge d a
$$

which is independent of the metric tensor as it should be. The phase space variables of the gauge field are $a_{1}(x)$ and $a_{2}(x) . a_{0}(x)$ is considered as a Lagrange multiplier. To quantize this system, we require the equal-time commutation relations as

$$
\begin{aligned}
& {\left[a_{1}(x), a_{2}\left(x^{\prime}\right)\right]=i \frac{\hbar}{\mu} \delta^{(2)}\left(\vec{x}-\vec{x}^{\prime}\right)} \\
& {\left[a_{i}(x), a_{0}\left(x^{\prime}\right)\right]=0}
\end{aligned}
$$

and impose the Lorentz gauge fixing condition,

$$
\nabla_{\mu} a^{\mu} \equiv \frac{1}{\sqrt{g}} \partial_{\mu} \sqrt{g} g^{\mu \nu} a_{\nu}=0
$$

To expand the gauge field in terms of modes, we use the Hodge decomposition (see Eq. (A11)), rather than using the vector harmonics directly [22]. The gauge field is written as

$$
a_{i}(x)=\partial_{i} \chi+\epsilon_{i j} g^{j l} \partial_{l} \xi
$$

$\chi$ and $\xi$ are mode expanded using spherical harmonics $Y_{l m}(\Omega)$,

$$
\begin{aligned}
& \chi(x)=-\frac{i}{\sqrt{2}} \sum_{l, m}^{\prime}\left[e^{-i \omega_{l} t} Y_{l m}(\Omega) \chi_{l m}-e^{i \omega_{l} t} Y_{l m}^{*}(\Omega) \chi_{l m}^{+}\right] \\
& \xi(x)=\xi_{B}(x)+\int d^{2} x^{\prime} \sqrt{g\left(x^{\prime}\right)} G\left(\vec{x}, \vec{x}^{\prime}\right) \zeta(x)
\end{aligned}
$$


where

$$
\zeta(x)=\frac{1}{\sqrt{2}} \sum_{l, m}^{\prime}\left[e^{-i \omega_{l} t} Y_{l m}(\Omega) \zeta_{l m}+e^{i \omega_{l} t} Y_{l m}^{*}(\Omega) \zeta_{l m}^{+}\right]
$$

Here $\omega_{l}=l(l+1)$ and the prime on the summation stands for the elimination of $l=0$ mode. $G\left(\vec{x}, \vec{x}^{\prime}\right)$ is the rotation invariant Green's function given in Eq. (A7). Each mode of the gauge field satisfies the commutation relations,

$$
\left[\chi_{l m}, \zeta_{l^{\prime} m^{\prime}}^{+}\right]=\left[\zeta_{l m}, \chi_{l^{\prime} m^{\prime}}^{+}\right]=\frac{\hbar}{\mu} \delta_{l l^{\prime}} \delta_{m m^{\prime}}
$$

and commutes with the rest so that Eq. (2.6) is satisfied. We added posssible background mode $\xi_{B}(x)$ in $\xi(x)$, which is commuting with all the other modes. According to the decomposition Eq. (2.8), we have

$$
\begin{aligned}
& \nabla_{i} a^{i} \equiv \frac{1}{\sqrt{g}} \partial_{i} \sqrt{g} g^{i j} a_{j}=-\nabla^{2} \chi \\
& \frac{1}{\sqrt{g}} b \equiv-\epsilon^{i j} \partial_{i} a_{j}=-\nabla^{2} \xi .
\end{aligned}
$$

Therefore, the background mode $\xi_{B}$ can accommodate a constant magnetic flux. $a_{0}(x)$ can be written in consistency with the gauge fixing condition Eq. (2.7) as

$$
a_{0}(x)=\lambda+\frac{1}{\sqrt{2}} \sum_{l, m}^{\prime}\left[e^{-i \omega_{l} t} Y_{l m}(\Omega) \xi_{l m}+e^{i \omega_{l} t} Y_{l m}^{*}(\Omega) \xi_{l m}^{+}\right]
$$

$\lambda$ is a space-time independent mode, which is still to be gauge fixed. (We also remark that the $\chi$ mode in Eq (2.8) has residual gauge degree of freedom [18)

The relation between the gauge fields on either patch is easily seen if we note the property of $\chi$ and $\zeta$ in Eq. (2.9). Let us denote $\chi_{(N)}\left(P_{N}\right)$ and $\zeta_{(N)}\left(P_{N}\right)$ as the north-patch description of $\chi$ and $\zeta$ at $\Omega$ respectively on the sphere. On the south-patch, they are denoted as $\chi_{(S)}\left(P_{S}\right)$ and $\zeta_{(S)}\left(P_{S}\right)$. For the overlapping region, the projected point $P_{S}\left(=z_{S}\right)$ of $\Omega=(\theta, \phi)$ to the south-patch is to be identified as $\tilde{P}_{N}\left(=-1 / z_{N}\right)$ on the north-patch (FIG.Q); $P_{S} \equiv \tilde{P}_{N}$ on the north patch for the overlapping region. Then $\tilde{P}_{N}$ becomes the projected point of $\tilde{\Omega}=(\pi-\theta, \pi-\phi)$. Noting that $Y_{l m}(\tilde{\Omega})=(-1)^{l} Y_{l m}^{*}(\Omega)=(-1)^{l+m} Y_{l,-m}(\Omega)$ we re-express $\chi_{(S)}\left(\tilde{P}_{N}\right)$ and $\zeta_{(S)}\left(\tilde{P}_{N}\right)$ as 


$$
\begin{aligned}
& \chi_{(S)}\left(\tilde{P}_{N}\right)=-\frac{i}{\sqrt{2}} \sum_{l, m}^{\prime}\left[e^{-i \omega_{l} t} Y_{l m}(\Omega)(-1)^{l+m} \chi_{(S) l,-m}-e^{i \omega_{l} t} Y_{l m}^{*}(\Omega)(-1)^{l+m} \chi_{(S) l,-m}^{+}\right] \\
& \zeta_{(S)}\left(\tilde{P}_{N}\right)=\frac{1}{\sqrt{2}} \sum_{l, m}^{\prime}\left[e^{-i \omega_{l} t} Y_{l m}(\Omega)(-1)^{l+m} \zeta_{(S) l,-m}+e^{i \omega_{l} t} Y_{l m}(\Omega)(-1)^{l+m} \zeta_{(S) l,-m}^{+}\right]
\end{aligned}
$$

With the identification,

$$
\begin{aligned}
& \chi_{(N) l m}=(-1)^{l+m} \chi_{(S) l,-m}, \quad \chi_{(N) l m}^{+}=(-1)^{l+m} \chi_{(S) l,-m}^{+} \\
& \zeta_{(N) l m}=(-1)^{l+m} \zeta_{(S) l,-m}, \quad \zeta_{(N) l m}^{+}=(-1)^{l+m} \zeta_{(S) l,-m}^{+},
\end{aligned}
$$

we have

$$
\chi_{(S)}\left(\tilde{P}_{N}\right)=\chi_{(N)}\left(P_{N}\right), \quad \zeta_{(S)}\left(\tilde{P}_{N}\right)=\zeta_{(N)}\left(P_{N}\right) .
$$

The sign reversal of $m$ arises because the rotation around $\hat{k}$ on one patch corresponds to the counter-rotation in the other. (See FIG.11). Representing the gauge field in complex notation as

$$
a=a_{1}-i a_{2}=2 \partial(\chi-i \xi), \quad \bar{a}=a_{1}+i a_{2}=2 \bar{\partial}(\chi+i \xi)
$$

we have the relation in the absence of the background mode $\left(\xi_{B}=0\right)$,

$$
a_{(N)}\left(P_{N}\right)=z_{S}^{2} a_{(S)}\left(\tilde{P}_{N}\right)
$$

using $\partial_{(N)}=z_{S}^{2} \partial_{(S)}$. The presence of background mode will, however, change the relation in Eq.(2.17), which will be important in the presence of matter field.

\section{GAUGED NON-RELATIVISTIC MATTER FIELD}

Let us couple the Chern-Simons gauge field to a non-relativistic fermionic matter field $\psi(x)$. Its action is given (on the north-patch) as

$$
S_{m}=\int d^{3} x \sqrt{g} \psi^{+} i \hbar D_{0} \psi-\frac{1}{2 m} \int d^{3} x\left(i \hbar D_{i} \psi\right)^{*}\left(i \hbar D_{i} \psi\right)
$$

in addition to the gauge part $S_{g}$ in Eq. (2.3). Here $i \hbar D_{\mu} \psi \equiv\left(i \hbar \partial_{\mu}-\frac{e}{c} a_{\mu}\right) \psi$. The matter field satisfies the equal-time anti-commutation relation, 


$$
\begin{aligned}
\left\{\psi(x), \psi^{+}\left(x^{\prime}\right)\right\} & =\frac{1}{\sqrt{g}} \delta^{(2)}\left(\vec{x}-\vec{x}^{\prime}\right) \\
\left\{\psi(x), \psi\left(x^{\prime}\right)\right\} & =0
\end{aligned}
$$

The hamiltonian is given as

$$
H=\int d^{2} x\left\{\mu a_{0} \Gamma+\frac{1}{2 m}\left(i \hbar D_{i} \psi\right)^{*}\left(i \hbar D_{i} \psi\right)\right\}
$$

whose form is similar to that on a plane. $\Gamma$ is the gauge generator given as

$$
\Gamma=b+\frac{e}{\mu c} \sqrt{g} J_{0}
$$

where $\sqrt{g} J_{0}$ is the number density operator, $J_{0}=\psi^{+} \psi$.

Since the gauge field has no dynamics, we may decouple the gauge field from the matter field by solving the constraint and get the effective hamiltonian. Instead of solving the constraint directly, we re-express the hamiltonian and the gauge generator in term of canonically transformed new fields as done in Ref. [8,18]

$$
\begin{aligned}
\Gamma & =b^{(1)}(x)+\frac{e}{\mu c} \frac{Q}{4 \pi} \sqrt{g(x)} \\
H & =\int d^{2} x\left\{\mu a_{0} \Gamma+\frac{1}{2 m}\left(i \hbar D_{i}^{(1)} \psi^{(1)}\right)^{*}\left(i \hbar D_{i}^{(1)} \psi^{(1)}\right)\right\} .
\end{aligned}
$$

where $Q$ is the number operator, $Q=\int d^{2} x \sqrt{g(x)} J_{0}(x)$. Here the old fields are related with the new ones as

$$
\begin{aligned}
& a_{i}(x)=V_{1}(t) a_{i}^{(1)}(x) V_{1}(t)^{+}=a_{i}^{(1)}(x)-\frac{e}{\mu c} e_{i j} \partial_{j} \int d^{2} x^{\prime} \sqrt{g\left(x^{\prime}\right)} G\left(\vec{x}, \vec{x}^{\prime}\right) J_{0}\left(x^{\prime}\right) \\
& \psi(x)=V_{1}(t) \psi^{(1)}(x) V_{1}^{+}(t)=\psi^{(1)}(x) \exp \left\{-i \frac{e}{\hbar c} \int d^{2} x^{\prime} G\left(\vec{x}, \vec{x}^{\prime}\right) \partial_{i}^{\prime} a_{i}^{(1)}\left(x^{\prime}\right)\right\}
\end{aligned}
$$

where

$$
V_{1}(t)=\exp \left\{i \frac{e}{\hbar c} \iint d^{2} x d^{2} x^{\prime} \sqrt{g(x)} J_{0}(x) G\left(\vec{x}, \vec{x}^{\prime}\right) \partial_{i}^{\prime} a_{i}^{(1)}\left(x^{\prime}\right)\right\}
$$

The hamiltonian $H$ is normal-ordered such that $\psi^{(1)+}$ lies in front of the effective covariant derivative $D_{i}^{(1)}$. More explicitly,

$$
\left(i \hbar D_{i}^{(1)} \psi^{(1)}\right)^{*} \equiv \psi^{(1)^{+}}\left(i \hbar D_{i}\right)^{+} \equiv-i \hbar \partial_{i} \psi^{(1)+}-\frac{e}{c} \psi^{(1)+} a_{i}^{[1] e f f}
$$


where

$a_{i}^{[1] e f f}(x)=e_{i j} \partial_{j}\left\{\frac{e}{4 \pi \mu c} \ln \left(1+\vec{x}^{2}\right)-\frac{e}{\mu c} \int d^{2} x \sqrt{g\left(x^{\prime}\right)} G\left(\vec{x}, \vec{x}^{\prime}\right) J_{0}\left(x^{\prime}\right)+\frac{1}{4 \pi} \int d^{2} x^{\prime} \ln \left(\vec{x}-\vec{x}^{\prime}\right)^{2} b^{(1)}\left(x^{\prime}\right)\right\}$

It should be noted that $b^{(1)}(x)$ rather than $a_{i}^{(1)}(x)$ appears in $a^{[1] e f f}(x)$, which is the chief merit of this transformation and the regularization $\left.e_{i j} \partial_{j} \ln \left(\vec{x}-\vec{x}^{\prime}\right)^{2}\right|_{\vec{x}=\vec{x}^{\prime}}=0$ is assumed.

Here, the gauge generator $\Gamma$ contains the number operator $Q$ as well as $b^{(1)}$. We have not transformed away $Q$ in the gauge generator $\Gamma$ as in the plane case due to possible conflict with $S O(3)$ rotation (Möbius transformation in complex notation) invariance of the system. To investigate the role of the rotation symmetry more clearly, let us introduce another canonical fields which get rid of $Q$ from $\Gamma$,

$$
\begin{aligned}
& a_{i}^{(1)}(x)=V_{2} a_{i}^{(2)}(x) V_{2}^{+}=a_{i}^{(2)}(x)-\frac{e Q}{4 \pi \mu c} e_{i j} \partial_{j} \ln \left(1+\vec{x}^{2}\right) \\
& \psi^{(1)}(x)=V_{2} \psi^{(2)}(x) V_{2}^{+}=\psi^{(2)}(x) \exp -i \eta_{0} .
\end{aligned}
$$

$V_{2}=\exp \left\{-i Q \eta_{0}\right\}$ and $\eta_{0}$ is a conjugate to $\xi_{0}$ of the background mode $\xi_{B}(x)$ defined as $\xi_{B}(x) \equiv \xi_{0} \frac{e}{4 \pi \mu c} \ln \left(1+\vec{x}^{2}\right)$

$$
\left[\eta_{0}, \xi_{0}\right]=i
$$

Then the hamiltonian and the gauge generator in Eq.(3.5) is written as

$$
\begin{aligned}
\Gamma & =b^{(2)} \\
H & =\int d^{2} x\left\{\mu a_{0} b^{(2)}+\frac{1}{2 m}\left(i \hbar D_{i}^{(2)} \psi^{(2)}\right)^{*}\left(i \hbar D_{i}^{(2)} \psi^{(2)}\right)\right\}
\end{aligned}
$$

where

$$
a_{i}^{[2] e f f}(x)=e_{i j} \partial_{j} \int d^{2} x^{\prime} \frac{\ln \left(\vec{x}-\vec{x}^{\prime}\right)^{2}}{4 \pi}\left\{-\frac{e}{\mu c} \sqrt{g\left(x^{\prime}\right)} J_{0}\left(x^{\prime}\right)+b^{(2)}\left(x^{\prime}\right)\right\}
$$

The gauge generator $b^{(2)}$ commutes with all the other fields in this representation and annihilates the physical Hilbert space. The gauge field $a_{i}^{(2)}(x)$ is therefore, decoupled from the matter sector and we may drop $b^{(2)}$ from the hamiltonian. The effective gauge field $a_{i}^{[2] e f f}$ turns out to be the solution of the constraint $\Gamma=0$ in Eq. (3.4). 
For the $S O(3)$ rotation invariant system, no point is regarded as special. Therefore, we have to introduce another equivalent hamiltonian on the south-patch. Adopt the same form of the hamiltonian on the south-patch as in Eq. (3.11), and we have a relation between the gauge field on each patch, which is a modified version of Eq. (2.17),

$$
a_{(N)}^{[2] e f f}\left(P_{N}\right)=z_{S}^{2}\left[a_{(S)}^{[2] e f f}\left(\tilde{P}_{N}\right)+\frac{e Q}{2 \pi \mu c} i \partial_{(S)} \ln \left(\frac{z_{S}}{\bar{z}_{S}}\right)\right]
$$

with the assumption that

$$
J_{0(N)}\left(P_{N}\right)=J_{0(S)}\left(\tilde{P}_{N}\right)
$$

which should be checked a posteriori. Considering the relation between the area element on each patch, $d^{2} x_{N}=d^{2} x_{S} /\left|z_{S}\right|^{4}$, we have the relation between matter fields up to a trivial constant phase factor,

$$
\psi_{(N)}\left(P_{N}\right)=\left(\frac{z_{S}}{\bar{z}_{S}}\right)^{\frac{\nu}{2} Q} \psi_{(S)}\left(\tilde{P}_{N}\right)
$$

where $\nu=\frac{e^{2}}{2 \pi \mu c^{2} \hbar}$. The number density satisfies the condition in Eq. (3.14). However, the presence of the number operator in the phase factor spoils this formalism since the identification in Eq. (3.15) will not respect the anti-commutation relations given in Eq. (3.2).

To avoid this obstruction, we present two ways out. One is to simply abandon $S O(3)$ but allows only $S O(2)$ rotation invariance around the axis through a point (e.g., the south-pole) which is to be excluded from the manifold. A sphere with the elimination of the one point is topologically equivalent to an infinite plane: We need only one coordinate-patch, which removes the subtlety. This system is best described with the hamiltonian in Eq. (3.11).

Another way is to maintain the $S O(3)$ invariance but changes the way of applying the gauge constraint on the physical state. The problem with the (anti-)commutation relation traces back to the presence of the number operator $Q$ attached to $S O(3)$ rotation noninvariant term $\ln \left(1+\vec{x}^{2}\right)$ in $a_{i}^{[2] e f f}(x)$. To cure this, we go back to the hamiltonian and gauge generator given in Eq.(3.5). Let us express $\Gamma$ as 


$$
\Gamma(x)=\left(b^{(1)}+\frac{e}{\mu c} \frac{\sqrt{g(x)}}{4 \pi} N\right)+\frac{e}{\mu c} \frac{\sqrt{g(x)}}{4 \pi}(Q-N)
$$

where $N$ is a $c$-number integer, which will be interpreted as the number of particles. Let us introduce similar canonical fields in Eq. (3.9).

$$
\begin{aligned}
& a_{i}^{(1)}(x)=V_{3} a_{i}^{(3)}(x) V_{3}^{+}=a_{i}^{(3)}(x)-\frac{e N}{4 \pi \mu c} e_{i j} \partial_{j} \ln \left(1+\vec{x}^{2}\right) \\
& \psi^{(1)}(x)=V_{3} \psi^{(3)}(x) V_{3}^{+}=\psi^{(3)}(x)
\end{aligned}
$$

where $V_{3}=\exp \left\{-i N \eta_{0}\right\}$. In terms of this new fields, we have the constraint

$$
\Gamma=b^{(3)}+\frac{e}{\mu c} \frac{\sqrt{g(x)}}{4 \pi}(Q-N)
$$

and the effective gauge term

$$
\begin{gathered}
a_{i}^{[3] e f f}(x)=e_{i j} \partial_{j}\left\{-\frac{e(N-1)}{4 \pi \mu c} \ln \left(1+\vec{x}^{2}\right)-\frac{e}{\mu c} \int d^{2} x^{\prime} \sqrt{g\left(x^{\prime}\right)} G\left(\vec{x}, \vec{x}^{\prime}\right) J_{0}\left(x^{\prime}\right)\right. \\
\left.+\frac{1}{4 \pi} \int d^{2} x^{\prime} \ln \left(\vec{x}-\vec{x}^{\prime}\right)^{2} b^{(3)}\left(x^{\prime}\right)\right\}
\end{gathered}
$$

We constrain the physical space as

$$
b^{(3)} \mid \text { phys }>=0 \quad \text { Q } \mid \text { phys }>=N \mid \text { phys }>
$$

Fixing the remnant gauge as $\lambda=0$ and dropping $b^{(3)}$, we have hamiltonian as

$$
H=\frac{1}{2 m}\left|\left(i \hbar \partial_{i}+e_{i j} \partial_{j}\left\{\frac{e^{2}(N-1)}{4 \pi \mu c^{2}} \ln \left(1+\vec{x}^{2}\right)+\frac{e^{2}}{\mu c^{2}} \int d^{2} x^{\prime} \sqrt{g\left(x^{\prime}\right)} G\left(\vec{x}, \vec{x}^{\prime}\right) J_{0}\left(x^{\prime}\right)\right\}\right) \psi^{(3)}\right|^{2}
$$

The difference of this hamiltonian from the one given in Eq. (3.11) is clear: $Q$ is replaced by a $c$-number $N-1$ (due to the normal-ordering of the operators, $\psi^{+}, Q$ and $\psi$ ). The equivalence between the matter fields on the north and the south patch is established by noting

$$
\psi_{(N)}\left(P_{N}\right)=\left(\frac{z_{S}}{\bar{z}_{S}}\right)^{\frac{\nu}{2}(N-1)} \psi_{(S)}\left(\tilde{P}_{N}\right)
$$


which respects the anti-commutation relation on either patch. The transition function in Eq. (3.22), however, has the cut from the south to north pole. To remove this cut, we need the Dirac quantization condition similarly appearing in the magnetic monopole analysis,

$$
(N-1) \nu=n
$$

where $n$ is an arbitrary integer. The factor $(N-1)$ rather than $N$ arises because the particle does not see its own flux. This condition is noted in the braid group analysis on the sphere 23]. By making the gauge sector look trivial (Eq. (3.20)) except the possible quantum holonomy, we have instead the explicit interaction term due to the magnetic monopole-like source inside the sphere. (See Eq. (3.21). If we go back to the original picture, $b^{(1)}$ in Eq. (3.16) is seen to possess the monopole source from the beginning proportional to the number of particles $N$.

One may also consider the case when $N$ is non-positive. For example, when $N=0$ the Hilbert space contains no particles at all in the above analysis. To make it physically interesting, one may introduce multi-species of the matter fields where each species has different coupling. We present the simplest choice, i.e., two species of matter fields with equal but opposite statistical coupling. Then the Hilbert space with $N=0$ consists of states with arbitrary number of particles and equal number of "anti-particles". The hamiltonian can be written as

$$
H=\frac{1}{2 m_{(+)}} \int d^{2} x\left|i \hbar D_{i(+)}^{(1)} \psi_{(+)}^{(1)}(x)\right|^{2}+\frac{1}{2 m_{(-)}} \int d^{2} x\left|i \hbar D_{i(-)}^{(1)} \psi_{(-)}^{(1)}(x)\right|^{2}
$$

The derivative operator is given as

$$
D_{i( \pm)}^{[1]}=i \hbar \partial_{i} \mp \frac{e}{\mu^{2} c} e_{i j} \partial_{j}\left\{\frac{1}{4 \pi} \ln \left(1+\vec{x}^{2}\right)-\int d^{2} x \sqrt{g\left(x^{\prime}\right)} G\left(\vec{x}, \vec{x}^{\prime}\right) J_{0}\left(x^{\prime}\right)\right\}
$$

where the charge density operator is defined as $J_{0}(x)=\psi_{(+)}^{+} \psi_{(+)}-\psi_{(-)}^{+} \psi_{(-)}$. The realtion of the matter field on each patch is given as

$$
\psi_{(N)( \pm)}\left(P_{N}\right)=\left(\frac{z_{S}}{\bar{z}_{S}}\right)^{\mp \frac{\nu}{2}} \psi_{(S)( \pm)}\left(P_{S}\right)
$$


which recalls the Dirac quantization condition in Eq. (3.23) with $N=0$;

$$
\nu=n
$$

Quite straight-forwardly, one can construct the system with negative $N$ introducing multispecies.

\section{ANYON EQUATION}

Suppose there are $N$ identical particles on a sphere. The dynamics of the particles can be described in two ways as given in Sec. III, which is distinguished by the rotation invariance. With only $S O(2)$ invariance, the particles obey the dynamics with the hamiltonian in Eq. (3.11). The statistical flux need not be quantized similarly as in the plane. On the other hand, $S O(3)$ invariance leads to the flux quantization Eq. (3.23), and the dynamics is described by the hamiltonian Eq. (3.21). The Schrödinger equation can be obtained from the Heisenberg equation for the corresponding matter field and its application to the $N$-body wave function. For the case with $S O(2)$ invariance, we obtain the Heisenberg equation,

$$
\begin{aligned}
i \hbar \frac{\partial \psi(x)}{\partial t} & =\frac{1}{\sqrt{g}}\left(i \hbar D_{i}^{(2)}\right)^{2} \psi(x) \\
& +\left(\frac{e}{c}\right)^{2} \int d^{2} x^{\prime} \psi^{+}\left(x^{\prime}\right) \psi\left(x^{\prime}\right)\left(R_{i}\left(\vec{x}^{\prime}, \vec{x}\right)\right)^{2} \psi(x) \\
& -\left(\frac{e}{c}\right) \int d^{2} x^{\prime} \psi^{+}\left(x^{\prime}\right)\left[\left(i \hbar D_{i}{ }^{(2)}\right)^{+}+i \hbar{D_{i}}^{(2)}\right] \psi\left(x^{\prime}\right) R_{i}\left(\vec{x}^{\prime}, \vec{x}\right) \psi(x)
\end{aligned}
$$

where $R_{i}\left(\vec{x}^{\prime}, \vec{x}\right) \equiv-\frac{e}{4 \pi \mu c} e_{i j} \partial_{j}^{\prime} \ln \left(\vec{x}-\vec{x}^{\prime}\right)^{2}$. Defining the $N$-particle wavefunction as

$$
\Phi(N)=<0\left|\prod_{p=1}^{N} \psi\left(x_{p}\right)\right| N>
$$

where $\mid N>$ is $N$-particle Heisenberg state, we have

$$
i \hbar \frac{\partial \Phi(N)}{\partial t}=\sum_{p=1}^{N} \frac{1}{2 m} \frac{1}{\sqrt{g\left(x_{p}\right)}}\left(i \hbar \partial_{i}^{(p)}+\mathcal{A}_{i}\left(x_{p}\right)\right)^{2} \Phi(N)
$$

where

$$
\mathcal{A}_{i}\left(x_{p}\right)=\frac{e^{2}}{4 \pi \mu c^{2}} e_{i j} \partial_{j}^{(p)} \ln \left\{\prod_{q(\neq p)}\left(\vec{x}_{p}-\vec{x}_{q}\right)^{2}\right\}
$$


$\mathcal{A}_{i}\left(x_{p}\right)$ satisfies the constraint,

$$
e_{i j} \partial_{j}^{(p)} \mathcal{A}_{j}\left(x_{p}\right)=-\frac{e^{2}}{4 \pi \mu c^{2}} \sum_{q(\neq p)} \delta^{(2)}\left(\vec{x}_{p}-\vec{x}_{q}\right)
$$

When there is a single particle $(N=1)$, the particle moves free $\left(\mathcal{A}_{i}=0\right)$. For $N \geq 2$, the wavefunction should satisfy the hard-core boundary condition between particles and also vanish at the south-pole. Since there is no statistical flux quantization, there exist cuts from the south-pole to the particle positions. In addition, one can transform the equation to a free form through a singular transformation,

$$
\tilde{\Phi}(N)=\prod_{p>q}\left(\frac{z_{p}-z_{q}}{\bar{z}_{p}-\bar{z}_{q}}\right)^{\frac{\nu}{2}} \Phi(N)
$$

where $z_{p}=x_{p}^{(1)}+i x_{p}^{(2)}$. $\tilde{\Phi}(N)$ is multi-valued under the exchange of particles whereas $\Phi(N)$ is anti-symmetric.

For the $S O(3)$ invariant case, using the wavefunction in Eq. (4.2) and Hamiltonian Eq. (3.21), we have the Schrödinger equation of the form in Eq. (4.3) where $\mathcal{A}_{i}\left(x_{p}\right)$ is now given as

$$
\mathcal{A}_{i}\left(x_{p}\right)= \begin{cases}0 & \text { for } N=1 \\ \frac{e^{2}}{\mu c^{2}} e_{i j} \partial_{j}^{(p)}\left\{\frac{N-1}{4 \pi} \ln \left(1+\vec{x}^{2}\right)+\ln \prod_{q(\neq p)} G\left(\vec{x}_{p}, \vec{x}_{q}\right)\right\} & \text { for } N \geq 2\end{cases}
$$

which remarkably turns out to be the same as the one given in Eq. (4.4). The distinctive point lies in that the statistical flux is quantized and one has only to apply the hard-core boundary condition between particles on the wavefunction. (There is no cut from the particle position to the south-pole.) The same Schrödinger equation is also obtained by Comtet et. al. in [19].

The wavefunction $\tilde{\Phi}(N)$ is easy to obtain when $\nu$ is an even integer, which is ordinary anti-symmetric product of the one-particle wavefunctions. However, for odd $\nu, \tilde{\Phi}$ is to be symmetric satisfying the hard-core condition, which is not obtained merely by symmetrization of the products of the one-particle wavefunctions. For general value of $\nu$, the wavefunction is not known yet. 
Schrödinger equation for two species can be obtained similarly. Using the hamiltonian in Eq. (3.24), one obtains the Heisenberg equation for the field,

$$
\begin{aligned}
& i \hbar \frac{\partial \psi_{( \pm)}(x)}{\partial t}= \pm \frac{1}{2 m_{( \pm)} \sqrt{g}}\left(i \hbar D_{i( \pm)}^{(1)}\right)^{2} \psi_{( \pm)}(x) \\
& +\left(\frac{e}{c}\right)^{2} \int d^{2} x^{\prime}\left[\frac{\psi_{(+)}^{+}\left(x^{\prime}\right) \psi_{(+)}\left(x^{\prime}\right)}{2 m_{(+)}}+\frac{\psi_{(-)}^{+}\left(x^{\prime}\right) \psi_{(-)}\left(x^{\prime}\right)}{2 m_{(-)}}\right]\left(K_{i}\left(\vec{x}^{\prime}, \vec{x}\right)\right)^{2} \psi_{( \pm)}(x) \\
& \mp\left(\frac{e}{2 m_{(+)} c}\right) \int d^{2} x^{\prime}\left[\psi_{(+)}^{+}\left(x^{\prime}\right)\left(i \hbar D_{i(+)}^{(1)}\right)^{+} \psi_{(+)}\left(x^{\prime}\right)+\psi_{(+)}^{+}\left(x^{\prime}\right)\left(i \hbar D_{i(+)}^{(1)} \psi_{(+)}\left(x^{\prime}\right)\right)\right] K_{i}\left(\vec{x}^{\prime}, \vec{x}\right) \psi_{( \pm)}(x) \\
& \pm\left(\frac{e}{2 m_{(-)} c}\right) \int d^{2} x^{\prime}\left[\psi_{(-)}^{+}\left(x^{\prime}\right)\left(i \hbar D_{i(-)}^{\prime(1)}\right)^{+} \psi_{(-)}\left(x^{\prime}\right)+\psi_{(-)}^{+}\left(x^{\prime}\right)\left(i \hbar D_{i(-)}^{\prime(1)} \psi_{(-)}\left(x^{\prime}\right)\right)\right] K_{i}\left(\vec{x}^{\prime}, \vec{x}\right) \psi_{( \pm)}(x)
\end{aligned}
$$

where $i \hbar D_{i}^{(1)} \equiv-i \hbar \partial_{i}-\frac{e}{c} a_{i}^{[1] e f f}$ and $K_{i}\left(\vec{x}^{\prime}, \vec{x}\right) \equiv-\frac{e}{4 \pi \mu c} e_{i j} \partial_{j}^{\prime} G\left(\vec{x}, \vec{x}^{\prime}\right)$. Defining the $N$-particle wavefunction as

$$
\Phi(N ; N) \equiv<0\left|\prod_{p=1}^{N} \psi_{(+)}\left(\vec{x}_{p}\right) \psi_{(-)}\left(\vec{y}_{p}\right)\right| N ; N>,
$$

we have the Schrödinger equation for $\Phi(N ; N)$ as follows.

$$
\begin{aligned}
i \hbar \frac{\partial \Phi(N ; N)}{\partial t}=\sum_{p=1}^{N} & \left\{\frac{1}{2 m_{(+)}} \frac{1}{\sqrt{g\left(x_{p}\right)}}\left(i \hbar \partial_{i}^{x_{p}}+\mathcal{A}_{i}\left(x_{p}\right)\right)^{2}\right. \\
& \left.+\frac{1}{2 m_{(-)}} \frac{1}{\sqrt{g\left(y_{p}\right)}}\left(i \hbar \partial_{i}^{y_{p}}+\mathcal{B}_{i}\left(y_{p}\right)\right)^{2}\right\} \Phi(N ; N)
\end{aligned}
$$

where

$$
\begin{aligned}
& \mathcal{A}_{i}\left(x_{p}\right)=\frac{e^{2}}{4 \pi \mu c^{2}} e_{i j} \partial_{j}^{x_{p}} \ln \frac{1}{\left(\vec{x}_{p}-\vec{y}_{p}\right)^{2}}\left\{\prod_{q(\neq p)} \frac{\left(\vec{x}_{p}-\vec{x}_{q}\right)^{2}}{\left(\vec{x}_{p}-\vec{y}_{q}\right)^{2}}\right\} \\
& \mathcal{B}_{i}\left(y_{p}\right)=\frac{e^{2}}{4 \pi \mu c^{2}} e_{i j} \partial_{j}^{y_{p}} \ln \frac{1}{\left(\vec{x}_{p}-\vec{y}_{p}\right)^{2}}\left\{\prod_{q(\neq p)} \frac{\left(\vec{y}_{p}-\vec{y}_{q}\right)^{2}}{\left(\vec{y}_{p}-\vec{x}_{q}\right)^{2}}\right\}
\end{aligned}
$$

One can put the Schrödinger equation in a free form if one makes a singular gauge transform,

$$
\tilde{\Phi}(N ; N)=\left[\left(\prod_{p, q} \frac{\bar{z}_{p}-\bar{w}_{q}}{z_{p}-w_{q}}\right)\left(\prod_{p>q} \frac{\left(z_{p}-z_{q}\right)\left(w_{p}-w_{q}\right)}{\left(\bar{z}_{p}-\bar{z}_{q}\right)\left(\bar{w}_{p}-\bar{w}_{q}\right)}\right)\right]^{\frac{\nu}{2}} \Phi(N ; N)
$$

where $z_{p}=x_{p}^{(1)}+i x_{p}^{(2)}$ and $w_{p}=y_{p}^{(1)}+i y_{p}^{(2)} . \tilde{\Phi}(N ; N)$ may change its statistics when two particles of the same species interchange each other by the factor $(-1)^{\nu}$ which is \pm 1 according to the Dirac quantization condition in Eq. (3.27). 


\section{EXTERNAL MAGNETIC SOURCE}

Suppose one introduces external magnetic source, whose field is constant on the surface and is perpendicular to the surface, magnetic monopole. Then the hamiltonian in Eq. (3.1) is modified such that the gauge field is replaced by $a_{i}+A_{i}^{e x t}$ where $A_{i}^{e x t}=\frac{\phi}{4 \pi} e_{i j} \partial_{i} \ln \left(1+\vec{x}^{2}\right)$ (or $\left.b^{\text {ext }}=\frac{\sqrt{g}}{4 \pi} \phi\right)$. For the $S O(3)$ invariance case, one can re-express the hamiltonian as in Eq. (3.21), where the covariant derivative is shifted by $-\frac{e}{c} A_{i}^{e x t}$. The compatibility of the two patch description leads to the Dirac quantization condition.

$$
(N-1) \nu-k=m
$$

where $k=\frac{e \phi}{h c}$.

Also the Schrödinger equation for $N$ particles is given as in Eq. (4.3) where $\mathcal{A}_{i}\left(x_{p}\right)$ is replaced by $\mathcal{A}_{i}\left(x_{p}\right)-\frac{e \phi}{4 \pi c} e_{i j} \partial_{j} \ln \left(1+\vec{x}_{p}^{2}\right)$, as is commonly found in the literature. In case $k$ is strong enough such that $m \leq 0$, one can easily construct the ground states and find their degeneracy. Define

$$
i \hbar \mathcal{D}=i \hbar\left(\mathcal{D}_{1}-i \mathcal{D}_{2}\right), \quad i \hbar \overline{\mathcal{D}}=i \hbar\left(\mathcal{D}_{1}+i \mathcal{D}_{2}\right)
$$

we have the Schrödinger equation for $N$ particles

$$
i \hbar \frac{\partial \Phi(N)}{\partial t}=\frac{\hbar^{2}}{2 m} \sum_{p=1}^{N} \frac{1}{\sqrt{g\left(x_{p}\right)}}\left(-\mathcal{D} \overline{\mathcal{D}}\left(x_{p}\right)+\frac{k}{2} \sqrt{g\left(x_{p}\right)}\right) \Phi(N) .
$$

The ground state is obtained [24] when $\overline{\mathcal{D}}\left(x_{p}\right) \Phi(N)=0$ whose solution has the form,

$\Phi(N)= \begin{cases}\prod_{p<q}\left(z_{p}-z_{q}\right) \prod_{p<q}\left|z_{p}-z_{q}\right|^{\nu} \prod_{p}\left(1+\left|z_{p}\right|^{2}\right)^{-\frac{k}{2}} S(z) ; & 0 \leq \frac{\nu}{2}<1 \\ \prod_{p<q}\left(\bar{z}_{p}-\bar{z}_{q}\right)^{(2 r+1)} \prod_{p<q}\left|z_{p}-z_{q}\right|^{(\nu-4 r-2)} \prod_{p}\left(1+\left|z_{p}\right|^{2}\right)^{-\frac{k}{2}} S(z) ; & 2 r+1 \leq \frac{\nu}{2}<2 r+3\end{cases}$

where $r$ is a non-negative integer. $S(z)$ is a symmetric holomorphic polynomial with degree $P_{s}$. The normalizability of $\Phi(N)$ restricts $S(z)$ : The power of $\left|z_{p}\right|$ should satisfy

$$
\begin{cases}(N-1)(\nu+1)-k+P_{s} \leq 0 ; & 0 \leq \frac{\nu}{2}<1 \\ (N-1)(\nu-2 r-1)-k+P_{s} \leq 0 ; & 2 r+1 \leq \frac{\nu}{2}<2 r+3\end{cases}
$$


When $S(z)=1$ and $N \rightarrow \infty$, filling factor $\alpha \equiv N / k$ is given as

$$
\alpha= \begin{cases}\frac{1}{\nu+1} ; & 0 \leq \frac{\nu}{2}<2 \\ \frac{1}{\nu-(2 r+1)} ; & 2 r+1 \leq \frac{\nu}{2}<2 r+3 .\end{cases}
$$

(For the bosonic wavefunction, $\Phi(N)$ in Eq. (5.4) is to be symmetrized and the corresponding filling factor is to be modified from Eq. (5.5).)

The Schrödinger equation becomes the ordinary monopole system after the singular gauge transformation

$$
\begin{aligned}
i \hbar \frac{\partial \tilde{\Phi}(N)}{\partial t} & =\frac{1}{2 m} \sum_{p=1}^{N} \frac{1}{\sqrt{g\left(x_{p}\right)}}\left(i \hbar \partial_{i}^{(p)}-\frac{e}{c} A_{i}^{e x t}\left(x_{p}\right)\right)^{2} \tilde{\Phi}(N) \\
& =\frac{\hbar^{2}}{2 m} \sum_{p=1}^{N}\left\{\frac{1}{2}\left(L_{p+} L_{p-}+L_{p-} L_{p+}\right)+\left(L_{p 3}\right)^{2}-\left(\frac{k}{2}\right)^{2}\right\} \tilde{\Phi}(N)
\end{aligned}
$$

where $L_{p+}=-\bar{\partial}^{(p)}-z_{p}^{2} \partial^{(p)}+\frac{k}{2} z_{p}, L_{p-}=\partial^{(p)}+\bar{z}_{p}^{2} \bar{\partial}^{(p)}+\frac{k}{2} \bar{z}_{p}, L_{p 3}=z_{p} \partial^{(p)}-\bar{z}_{p} \bar{\partial}^{(p)}-\frac{k}{2} z_{p} . L_{p}{ }^{\prime} \mathrm{s}$ are $s u(2)$ generators [25],

$$
\begin{aligned}
{\left[L_{p+}, L_{q-}\right] } & =2 L_{p 3} \delta_{p q} \\
{\left[L_{p 3}, L_{q \pm}\right] } & = \pm L_{p \pm} \delta_{p q} .
\end{aligned}
$$

Without the statistical interaction, one can obtain the spectrum and degeneracy using the $s u(2)$ representation. On the other hand, since $\tilde{\Phi}$ is multi-valued due to the statistical interaction, the usefulness of $s u(2)$ algebraic structure is not clear. Although the ground state solution in Eq. (5.4) is the eigenstate of $\sum_{p} L_{p 3}$ and $H$, it is not annihilated by $L_{p-}$ ( $\sum_{p} L_{p-}$ does annihilate the state but it does not help much).

\section{SUMMARY AND DISCUSSION}

We have investigated the role of the rotation symmetry to the anyon equation employing the canonically transformed new fields in the CS hamiltonian. To maintain the $S O(3)$ rotation invariance, one has to restrict the Hilbert space to the one with definite charge. The consistency for the two patch description requires the CS coefficient quantized, which has the same consequencies as in the effect due to the monopole source. 
On the other hand, if one allows $S O(2)$ rotation invariance only, then one may eliminate one point from the sphere and the manifold is topologically equivalent to the infinite plane. The Hilbert space need not be restricted to the definite charge states and the CS coefficient does not have to be quantized. The Schrödinger equation is, however, remarkably the same for both the cases while the boundary conditions to be applied on the wavefunction differ.

The analysis on the sphere is equally applicable to the case on a torus. The appearance of non-commuting zero-modes of the gauge field due to the harmonic form makes the analysis complicated in the intermediate steps. However, once we get the effective gauge terms for the matter field, the analysis is straight-forward.

If one identifies the fundamental domain of the torus as the rectangular lattice (see FIG.3), then the CS theory can be made periodic both in the direction: The translation operators commute each other. In this case, there is no Dirac quantization condition. On the other hand, suppose one has the freedom to choose a parallelogram (see FIG.(1) as the unit cell (modular transformation) [26], then the translation operators do not commute each other. The trouble lies in the effective gauge term, which is given as

$$
A_{i}^{e f f}(x)=-\frac{e}{\mu c} \epsilon_{i j} \partial_{j} \int d \vec{y} G_{p}(\vec{x}, \vec{y}) J_{0}(y)-\frac{e}{\mu c} \epsilon_{i j} \partial_{j} \int d \vec{y} G_{n p}(\vec{x}, \vec{y}) J_{0}(y)
$$

where

$$
\begin{aligned}
G_{p}(\vec{x}, \vec{y}) & =\frac{1}{4 \pi} \ln \left|\frac{\theta_{1}(z \mid \tau)}{\theta_{1}^{\prime}(0 \mid \tau)}\right|^{2}-\frac{\left(x_{2}-y_{2}\right)^{2}}{2 L_{1} L_{2}} \\
G_{n p}(\vec{x}, \vec{y}) & =\frac{\left(x_{2}-y_{2}\right)^{2}}{2 L_{1} L_{2}} .
\end{aligned}
$$

(Here we follow the same notation given in [18].) $G_{p}$ is periodic under the translation along the modular transformed unit cell, whereas $G_{n p}$ is not. The translation results in the additional term depending on the total charge operator $Q$,

$$
A_{i}^{e f f}(x) \rightarrow \begin{cases}A_{i}^{e f f}(x) & \text { when } x^{1} \rightarrow x^{1}+L_{1} \\ A_{i}^{e f f}(x)-\frac{e}{\mu c} \frac{Q}{L_{1}} \delta_{i 1} & \text { when } x^{2} \rightarrow x^{2}+L_{2} .\end{cases}
$$

This result is similar to the one in sphere Eq. (3.13). 
To fix this, one can have two choices. One is to abandon the modular invariance: One requires the square lattice as the fundamental domain and gives the periodic condition at the edge. The other way is to maintain the modular invariance but restricts the Hilbert space to the one with definite charges. Following the similar produre done on the sphere, one can obtain the effective gauge term,

$$
A_{i}^{e f f}(x)=-(N-1) \tilde{x}_{i}-\frac{e}{\mu c} \epsilon_{i j} \partial_{j} \int d \vec{y} G_{p}(\vec{x}, \vec{y}) J_{0}(y)+\int d \vec{y} \tilde{y}_{i} J_{0}(y)
$$

where $\tilde{x}_{1}=\frac{e}{\mu c} \frac{x^{2}}{L_{1} L_{2}}$ and $\tilde{x}_{2}=0$. In this case, we have

$$
A_{i}^{e f f}(x) \rightarrow \begin{cases}A_{i}^{e f f}(x) & \text { when } x^{1} \rightarrow x^{1}+L_{1} \\ A_{i}^{e f f}(x)-\frac{e(N-1)}{\mu c L_{1}} \delta_{i 1} & \text { when } x^{2} \rightarrow x^{2}+L_{2} .\end{cases}
$$

Then the field operator has the translation property

$$
\begin{aligned}
& \psi\left(\vec{x}+L_{2} \hat{e}_{2}\right)=\psi(\vec{x}) \exp \left\{\frac{i e^{2}(N-1) x^{1}}{\hbar \mu c^{2} L_{1}}\right\} \\
& \psi\left(\vec{x}+L_{1} \hat{e}_{1}\right)=\psi(\vec{x}) .
\end{aligned}
$$

The consistency condition requires the same quantization condition as in the sphere Eq. (3.23). The many-anyon Schrödinger equation on the torus obtained in 18 is also applied to this case. The boundary condition on the wavefunction is, however, to be assigned in accordance with the appropriate symmetry in accordance with Eq. (6.5).

Finally, it would be challenging to extend this analysis to the non-abelian CS theory and demonstrate the anyon equation obtained in [13] from the second-quantized field theory.

\section{ACKNOWLEDGMENTS}

The authors have benefit from the discussion with Dr. K. Cho. This work is supported in part by KOSEF No. 931-0200-030-2, by Basic Science Research Institute program (BSRI94-2434) Ministry of Education, and by SRC program through Seoul National University. 


\section{REFERENCES}

[1] J. Leinaas and J. Myrheim, Nuovo Cim. B 37, 1 (1977) ; G. Goldin, R. Menikoff and D. H. Sharp, J. Math. Phys. 22, 1664 (1981) ; F. Wilczeck, Phys. Rev. Lett. 49, 957 (1982) ; Y. S. Wu, Phys. Rev. Lett. 52, 2103 (1984).

[2] For a review and references, see S. M. Girvin and R. Prange, The Quantum Hall Effect (Springer, New York, 1990).

[3] F. D. M. Haldane, Phys. Rev. Lett. 66, 1529 (1991).

[4] D. Arovas, J. R. Schrieffer and F. Wilczek, Phys. Rev. Lett. 53, 722 (1984).

[5] A. S. Goldhaber, R. Mackenzie and F. Wilczek, Mod. Phys. Lett. A 4, 21 (1989) ; T. H. Hansson, M. Roček, I. Zahed and S. C. Zhang, Phys. Lett. B214, 475 (1988).

[6] G. W. Semenoff, Phys. Rev. Lett. 61. 517 (1988) ; G. W. Semenoff, P. Sodano and Y. S. Wu, ibid. 62, 715 (1989) ; G. W. Semenoff and P. Sodano, Nucl. Phys. B 328, 753 (1989).

[7] T. Matsuyama, Phys. Lett. B228, 99 (1989) ; Phys. Rev. D 15, 3469 (1990) ; D. Boyanovsky, E. Newman and C. Rovelli, Phys. Rev. D 45, 1210 (1992) ; R. Banerjee, Phys. Rev. Lett. 69, 17 (1992) ; Nucl. Phys. B 390, 681 (1993).

[8] M. S. Swanson, Phys. Rev. D 42, 552 (1990); K. H. Cho and C. Rim, Int. J. Mod. Phys. A 7, 381 (1992).

[9] R. Jackiw and V. P. Nair, Phys. Rev. D 43, 1933 (1991): M. S. Plushchay, Phys. Lett. B262, 71 (1991).

[10] R. Jackiw and S. Templeton, Phys. Rev. D 23, 2291 (1981): J. Schonfeld, Nucl. Phys. B 185, 157 (1981): S. Deser, R. Jackiw and S. Templeton, Phys. Rev. Lett. 48, 975 (1982); Ann. Phys. (N. Y.) 140, 372 (1982): C. Hagen, Ann. Phys. (N. Y.) 157, 342 (1984) ; Phys. Rev. D 31, 2135 (1985). 
[11] R. Jackiw and S. -Y. Pi, Phys. Rev. D 42, 3500 (1990): C. Kim, C. Lee, P. Ko, B. -H. Lee and H. Min, Phys. Rev. D 48, 1821 (1993).

[12] E. Witten, Comm. Math. Phys. 121, 351 (1989).

[13] E. Verlinde, in Modern Quantum Field Theory (World Scientific, Singapore, 1991): X. G. Wen, Phys. Rev. Lett. 66, 802 (1991): G. Moore and N. Read, Nucl. Phys. B360, 362 (1991): T. Lee and P. Oh, Phys. Rev. Lett., 72, 1141 (1994).

[14] G. V. Dunne, R. Jackiw, and C. A. Trugenberger, Ann. Phys. (NY) 194, 197 (1989); G. Zemba, Int. J. Mod. Phys. A5, 559 (1990).

[15] Y. Hosotani, Phys. Rev. Lett. 62, 2785 (1989) ; C. -L. Ho and Y. Hosotani, Int. J. Mod. Phys. A 7, 5797 (1992) ; Phys. Rev. Lett. 70, 1360 (1993).

[16] A. Fayyazuddin, Nucl. Phys. B 401, 644 (1993).

[17] C. R. Hagen and E. C. G. Sudarshan, Phys. Rev. Lett. 64, 1690 (1990); preprint hepth/930163; Y. Hosotani, Phys. Rev. Lett. 64, 1691 (1990); C. -L. Ho and Y. Hosotani, preprint hep-th/9403156.

[18] K. Cho and C. Rim, preprint snutp93-96 (hep-th/9312204), to appear in Phys. Rev. D. (1994).

[19] K. Lee, preprint BU 89-28 (1989); A. Comtet, J. McCabe and S. Ouvry, Phys. Rev. D 45, 709 (1992).

[20] R. Iengo and K. Lechner, Phys. Rep. 213, 179 (1992).

[21] For review and references, see M. Nakahara, Geometry, Topology and Physics (Adam Hilger, Bristol, 1990).

[22] A. R. Edmonds, Angular Momentum in Quantum Mechanics (Princeton Press, NJ, 1974). 
[23] D. J. Thouless and Y. -S. Wu, Phys. Rev. B31, 1191 (1985).

[24] D. Li and S. Ouvry, preprint cond-mat/9403074.

[25] T. T. Wu and C. N. Yang, Nucl. Phys. B107, 365 (1976); G. Dunne, preprint CTP\#2015 (1991).

[26] K. Lechner, Phys. Lett. B273, 463 (1991).

\section{APPENDIX A:}

We summarize some of the useful formulae on a sphere. The line element on the sphere is written as

$$
\begin{aligned}
(d s)^{2} & =(d \theta)^{2}+\sin ^{2} \theta(d \phi)^{2} \\
& =\left(\frac{2}{1+\vec{x}^{2}}\right)^{2}\left[\left(d x^{1}\right)^{2}+\left(d x^{2}\right)^{2}\right]
\end{aligned}
$$

where $\Omega=(\theta, \phi)$ and $P_{N}=\left(x^{1}, x^{2}\right)$ according to FIG.11. This holds for the north-patch $(0 \leq \theta<\pi ;|\vec{x}|<\infty)$. Similar formula can be expressed for the south-patch. The invariant measure is given as

$$
d \tau=\sin \theta d \theta d \phi=\sqrt{g} d x^{1} d x^{2}
$$

where $g$ is the determinant of the metric in space 2 dimension,

$$
g_{i j}=-\left(\frac{2}{1+\vec{x}^{2}}\right)^{2} \delta_{i j}=-\sqrt{g} \delta_{i j}
$$

$\left(\delta_{11}=\delta_{22}=1, \delta_{12}=\delta_{21}=0\right)$. We add the negative sign to $g_{i j}$ to make it consistent with the 3 - $d$ metric whose signature is $(+,-,-)$. The 2 -d laplacian is given as

$$
\nabla^{2} \equiv-\frac{1}{\sqrt{g}} \partial_{i} \sqrt{g} g^{i j} \partial_{j}=\frac{1}{\sqrt{g}} \delta_{i j} \partial_{i} \partial_{j}=\left(\frac{1+\vec{x}^{2}}{2}\right)^{2}\left(\partial_{1}^{2}+\partial_{2}^{2}\right)
$$

The 2-d delta function is written as

$$
\delta^{(2)}\left(\Omega-\Omega^{\prime}\right)=\frac{1}{\sqrt{g}} \delta\left(x^{1}-x^{1 \prime}\right) \delta\left(x^{2}-x^{2 \prime}\right)=\frac{1}{\sqrt{g}} \delta^{(2)}\left(\vec{x}-\vec{x}^{\prime}\right)
$$


The Green's function on the sphere is defined as

$$
\nabla^{2} G\left(\vec{x}, \vec{x}^{\prime}\right)=\frac{1}{\sqrt{g}} \delta^{(2)}\left(\vec{x}-\vec{x}^{\prime}\right)-\frac{1}{4 \pi}
$$

where the constant term in the RHS is due to the lack of zero-mode contribution to the Green's function. Explicitly, $G\left(\vec{x}, \vec{x}^{\prime}\right)$ can be written as

$$
G\left(\vec{x}, \vec{x}^{\prime}\right)=\frac{1}{4 \pi} \ln \left\{\frac{\left(\vec{x}-\vec{x}^{\prime}\right)^{2}}{\left(1+\vec{x}^{2}\right)\left(1+\vec{x}^{\prime 2}\right)}\right\}
$$

We write down some of the properties of $G\left(\vec{x}, \vec{x}^{\prime}\right)$.

1. $G\left(\vec{x}, \vec{x}^{\prime}\right)$ is invariant under the rotation of the sphere, which corresponds to the Möbius transformation in the complex notation, $z=x^{(1)}+i x^{(2)} \rightarrow \frac{a z+b}{-b z+\bar{a}}$ where $a \bar{a}+b \bar{b} \neq 0$.

2. $\left(\partial_{1} \partial_{1}^{\prime}+\partial_{2} \partial_{2}^{\prime}\right) G\left(\vec{x}, \vec{x}^{\prime}\right)=-\delta^{(2)}\left(\vec{x}-\vec{x}^{\prime}\right)$, which is checked by explicit calculation.

3. $\int d^{2} x \sqrt{g(x)} G\left(\vec{x}, \vec{x}^{\prime}\right)=\int d^{2} x \sqrt{g(x)} G(\vec{x}, 0)=-1$ due to the rotation invariance.

4. $G\left(\vec{x}, \vec{x}^{\prime}\right)=G\left(\vec{x}^{\prime}, \vec{x}\right)$.

The gauge field on the sphere can be decomposed into the gradient part and the curl part as in the plane. For this purpose, we introduce form notation. The gauge one form is given as $a=a_{i} d x^{i}$. The measure in Eq. (A2) is expressed in two form and is given as $d \tau=\sqrt{g} d x^{1} \wedge d x^{2}$. In terms of anti-symmetric tensor $\epsilon_{i j}\left(\equiv \epsilon_{0 i j}\right)$, we can write this area form as $d \tau=\frac{1}{2} \epsilon_{i j} d x^{i} \otimes d x^{j}$, where $\epsilon_{i j}=\sqrt{|g|} e_{i j}$ and $e_{i j}$ is a (pseudo) invariant with $e_{12}=e^{12}=-e^{21}=-e_{21}=1$. The anti-symmetric tensor enables us to define the duality $($ Hodge $*$ ) transformation which transforms $r$-form into $2-r$ form,

$$
*\left(d x^{\mu_{1}} \wedge \cdots \wedge d x^{\mu_{r}}\right)=\frac{1}{(2-r) !} \epsilon_{\nu_{r+1} \cdots \nu_{m}}^{\mu_{1} \cdots \mu_{\mu}}\left(d x^{\nu_{r+1}} \wedge \cdots \wedge d x^{\nu_{m}}\right)
$$

Explicitly, $* 1=\sqrt{g} d x^{1} \wedge d x^{2}, * d x^{i}=\epsilon_{j}^{i} d x^{j}=g^{i j} \epsilon_{k j} d x^{j}=-e_{i j} d x^{j}, * \sqrt{g} d x^{1} \wedge d x^{2}=\sqrt{g} \epsilon^{12}=$ $e_{12}=1$.

The gauge one form is decomposed (Hodge decomposition) as

$$
a=d \chi+d^{+}(\xi d \tau)
$$


where $\chi$ and $\xi$ are zero-one form (function) and $d^{+}$is the adjoint operator defined as

$$
d^{+}(\xi d \tau)=-* d *(\xi d \tau)=-e_{i j}\left(\partial_{j} \xi\right) d x^{i}
$$

There is no harmonic form to the decomposition. In component notation, the gauge field is expressed as

$$
a_{i}=\partial_{i} \chi-e_{i j} \partial_{j} \xi=\partial_{i} \chi+\epsilon_{i j} g^{j l} \partial_{l} \xi
$$




\section{FIGURES}

FIG. 1. (a) North patch: $\Omega$ on the sphere is projected to $P_{N}$ on the plane and the north pole to $O$. The south pole is eliminated. (b) South patch: $\Omega$ is projected to $P_{S}$ and the south pole to $O$. The north-pole is eliminated.

FIG. 2. Identification of the north patch with the south.

FIG. 3. Rectangular unit cell as the fundamental domain of the torus.

FIG. 4. Two kinds of modular transformed unit cells. 


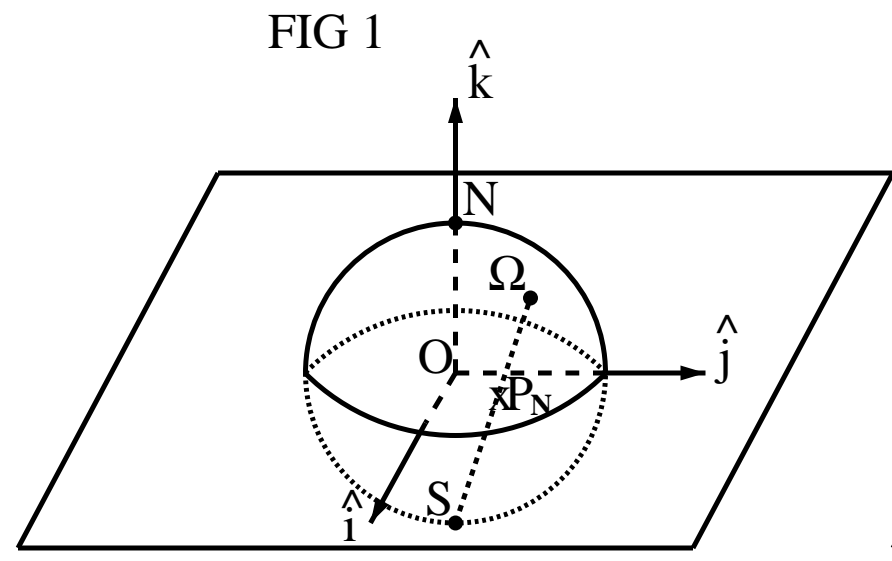

(a)

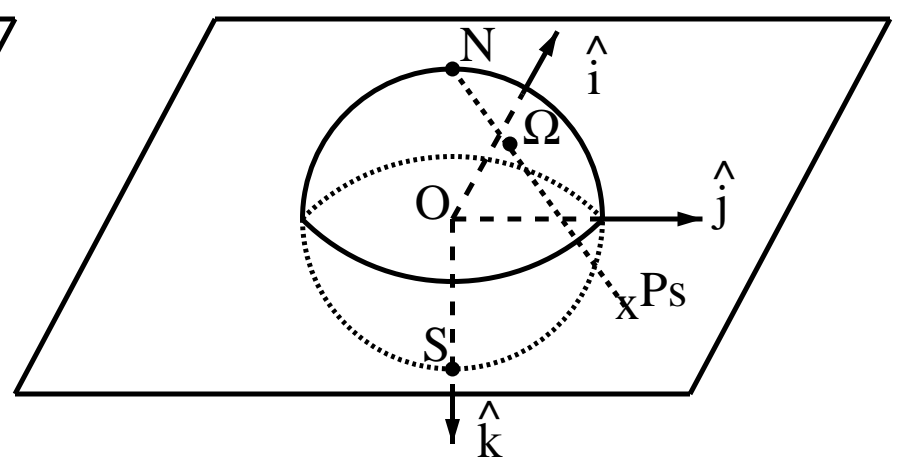

(b)

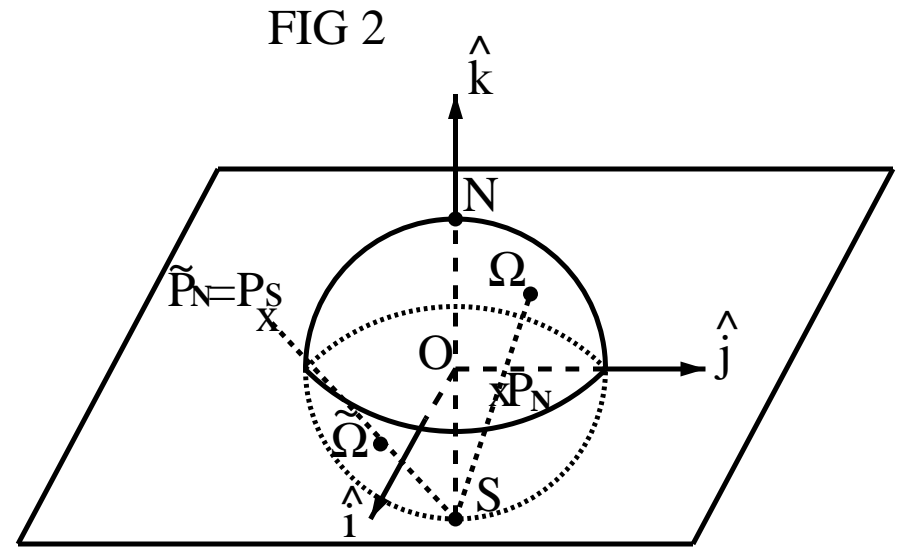


FIG 3

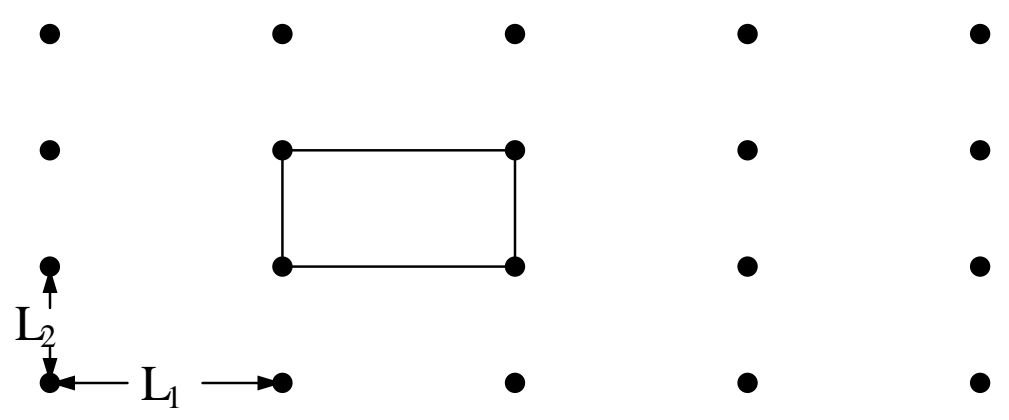

FIG 4
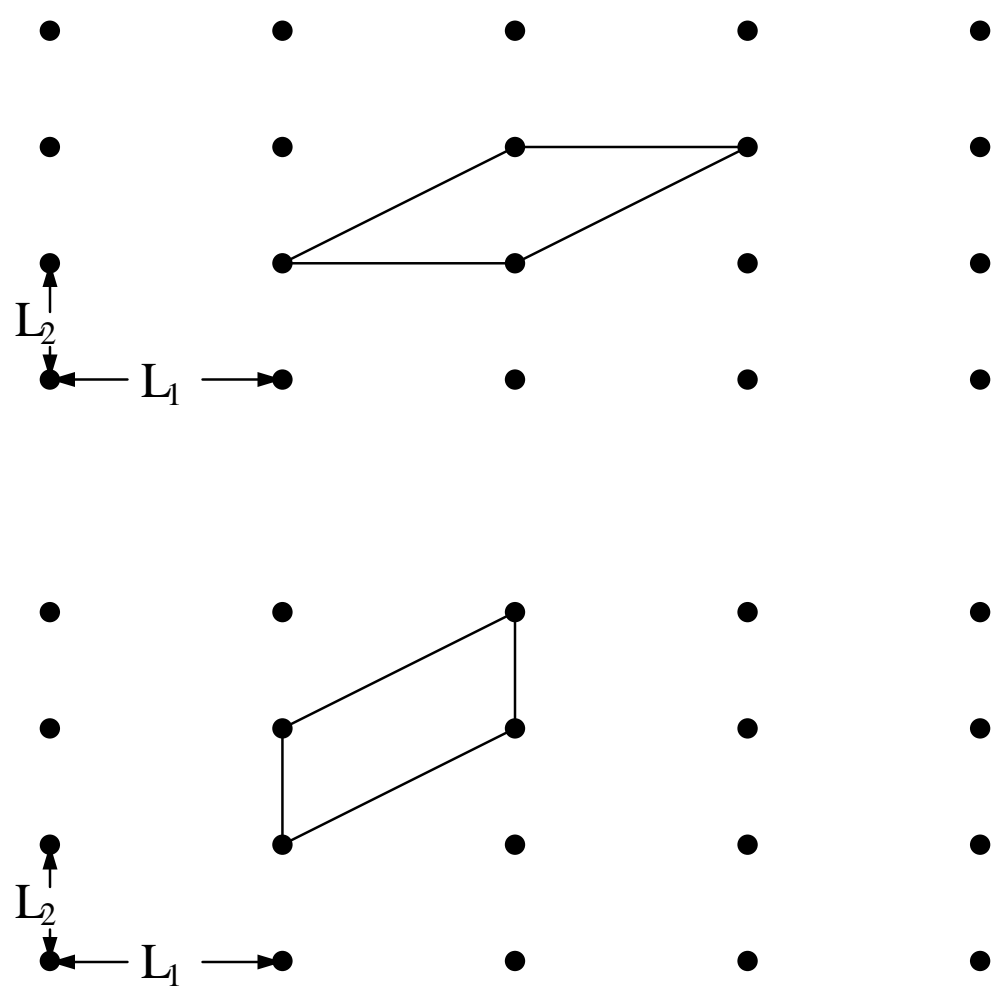